\title{
Effects of Stacking Fault Energy on Fundamental Deformation Modes in Single Crystalline Magnesium by Molecular Dynamics Simulations
}

\author{
Daisuke Matsunaka ${ }^{1,2, *}$, Yasuaki Ohnishi ${ }^{1}$ and Yoji Shibutani ${ }^{1,2}$ \\ ${ }^{1}$ Department of Mechanical Engineering, Osaka University, Suita 565-0871, Japan \\ ${ }^{2}$ Center for Atomic and Molecular Technologies, Osaka University, Suita 565-0871, Japan
}

\begin{abstract}
In order to investigate effects of stacking fault energies (SFEs) on fundamental deformation modes of slips and deformation twinnings in magnesium, we carried out molecular dynamics simulations of shear deformations for the deformation modes with two kinds of many-body interatomic potentials. The SFEs of the basal and second-pyramidal planes are lower for a generalized embedded atom method (GEAM) potential than for an embedded atom method (EAM) potential. While the basal slip quite easily occurs and the prism dislocation is activated, the first-pyramidal slip and the second-pyramidal slip are hard to be operated. However, for the GEAM simulations, the second-pyramidal slip was activated due to reduction of the second-pyramidal SFE. Additionally, the reduction of the SFEs suppresses nucleation of the $\{10 \overline{1} 1\}$ twin in the $b_{2}^{\{1011\}}$ shearing direction. The relative order of the other fundamental deformation modes in the critical shear stress is qualitatively maintained despite the reduction of the SFEs. [doi:10.2320/matertrans.MAW201311]
\end{abstract}

(Received May 1, 2013; Accepted August 30, 2013; Published October 11, 2013)

Keywords: magnesium, molecular dynamics simulations, stacking fault energy, deformation twinning

\section{Introduction}

Magnesium has been of increasingly interest from the engineering viewpoint, because of its low density and relatively high specific strength. ${ }^{1)}$ Despite intensive research efforts, there remain various problems to be overcome, e.g., low ductility and low toughness at room temperature. As for the former, five independent slip systems are necessary for the sufficient plastic deformability of polycrystals (that is the von Mises' criterion). Magnesium has only the major slip system of basal slip at room temperature, because the critical resolved shear stresses (CRSS) for the non-basal slip systems are much higher. Therefore, deformation twinning alternatively plays an important role in compensating the lack of the slip systems and maintaining a plastic flow of polycrystals. ${ }^{2-4)}$ There are two typical twinning modes for magnesium; $\{10 \overline{1} 2\}$ and $\{10 \overline{1} 1\}$ twinnings. The $\{10 \overline{1} 2\}$ twin is generated at an early stage of plastic deformation and its CRSS is only a few $\mathrm{MPa},{ }^{5)}$ comparable to the CRSS for the basal slip.

In $\mathrm{Mg}$ alloys, addition of rare earth elements in solid solution causes a significant increase in ductility. ${ }^{6-8)}$ A recent study has attributed the activation of $\langle c+a\rangle$ dislocations in $\mathrm{Mg}-\mathrm{Y}$ alloys to the intrinsic stacking faults of the basal plane. $\left.{ }^{9}\right)$ A further investigation is necessary to identify effects of solute atoms on the plastic properties in actual alloys.

A detailed understanding of the fundamental deformation modes of basal slip, prism slip, first-pyramidal slip, secondpyramidal slip, $\{10 \overline{1} 2\}$ deformation twinning, and $\{10 \overline{1} 1\}$ deformation twinning under a specified deformation condition is worthful to achieve the desirable plastic properties. In this study, we carry out molecular dynamics (MD) simulations of a hexagonal close-packed (hcp) magnesium atomic model under a simple shear deformation along the slip plane or the $K_{1}$ plane. By employing two different manybody interatomic potentials, effects of stacking fault energy (SFE) on the fundamental deformation modes are especially highlighted.

*Corresponding author, E-mail: matsunaka@mech.eng.osaka-u.ac.jp

\section{Methods}

In MD simulations, two kinds of many-body interatomic potentials, i.e., an embedded atom method (EAM) potential developed by Sun et al. ${ }^{10)}$ and a generalized embedded atom method (GEAM) potential by by Zhou et al. ${ }^{11)}$ were adopted to describe the interatomic interaction of magnesium. The physical and mechanical properties of the single crystal by EAM and GEAM potentials are summarized in Table 1. The lattice parameters and the elastic constants by both of the potentials are well consistent with the experimental values. ${ }^{12)}$ However, the SFEs of two basal intrinsic stacking faults, $I_{1}$ (stacking sequence ...ABABCBCB...) and $I_{2}$ (...ABCACAC...) and the SFE of the second-pyramidal plane by GEAM are lower than those by EAM. Comparing results obtained by the EAM and GEAM potentials, we intended to investigate effects of reduction of the SFEs on the fundamental deformation modes.

To realize a shear deformation simulation of each fundamental deformation mode, we prepared an atomic model where the slip plane or the $K_{1}$ plane was normal to the $z$-axis and the shearing direction was in the $x$ direction. The model size was $12.8 \mathrm{~nm} \times 11.1 \mathrm{~nm} \times 10.4 \mathrm{~nm}$ and the total number of atoms was approximately 64000. The top and bottom several atomic layers were fixed and the other surfaces were free. Shear deformation with respect to the slip plane or the $K_{1}$ plane was applied to the whole of the model with the strain rate of $10^{8} 1 / \mathrm{s}$. During the deformation, the temperature of the whole model was maintained at $1 \mathrm{~K}$ by scaling the velocities of atoms.

\section{Results and Discussions}

Based on the deformation manners, the slip modes are categorized into two types: one is the basal slip and the prism slip and the other is the first-pyramidal slip and the secondpyramidal slip. As shown in Fig. 1, the shear deformation of the $\{0001\}\langle 1 \overline{2} 10\rangle$ basal slip (see Fig. 1(b)) and the $\{10 \overline{1} 0\}\langle\overline{1} 2 \overline{1} 0\rangle$ prism slip (see Fig. 1(c)) gave rise to partial 
Table 1 Lattice parameters and elastic constants by the EAM and GEAM potentials and experimental values. The lattice constant, the $c / a$ ratio $(r=c / a)$, the elastic constants, the stacking fault energies of the $I_{1}$ and $I_{2}$ basal intrinsic stacking faults, $\gamma_{1}^{\mathrm{SF}}$ and $\gamma_{2}^{\mathrm{SF}}$, the stacking fault energies of the second-pyramidal stacking fault, $\gamma_{\{11 \overline{2} 2\}}^{\mathrm{SF}}$

\begin{tabular}{|c|c|c|c|c|c|c|c|c|c|c|}
\hline & $\begin{array}{c}a \\
{[\mathrm{~nm}]}\end{array}$ & $r$ & $\begin{array}{c}C_{11} \\
{[\mathrm{GPa}]}\end{array}$ & $\begin{array}{c}C_{33} \\
{[\mathrm{GPa}]}\end{array}$ & $\begin{array}{c}C_{44} \\
{[\mathrm{GPa}]}\end{array}$ & $\begin{array}{c}C_{12} \\
{[\mathrm{GPa}]}\end{array}$ & $\begin{array}{c}C_{13} \\
{[\mathrm{GPa}]}\end{array}$ & $\begin{array}{c}\gamma_{1}^{\mathrm{SF}} \\
{\left[\mathrm{mJ} / \mathrm{m}^{2}\right]}\end{array}$ & $\begin{array}{c}\gamma_{2}^{\mathrm{SF}} \\
{\left[\mathrm{mJ} / \mathrm{m}^{2}\right]}\end{array}$ & $\begin{array}{c}\gamma_{\{11 \overline{2} 2\}}^{\mathrm{SF}} \\
{\left[\mathrm{mJ} / \mathrm{m}^{2}\right]}\end{array}$ \\
\hline EAM & 0.318 & 1.63 & 69.0 & 70.8 & 12.7 & 25.3 & 15.9 & 22 & 44 & 188 \\
\hline GEAM & 0.320 & 1.63 & 55.9 & 69.4 & 13.8 & 28.7 & 20.2 & 2 & 4 & 152 \\
\hline Exp. ${ }^{12,13)}$ & 0.321 & 1.62 & 59.3 & 61.5 & 16.4 & 25.3 & 21.4 & - & 50 & - \\
\hline
\end{tabular}

dislocations from the free surfaces, and the trading partials subsequently moved. In Figs. 1(b) and 1(c), high-energy regions correspond to the dislocations and move parallel to the slip planes with shear deformations. This dislocation generation leads to the first peak observed in the shear stress and shear stress curves in Fig. 1(a). Recall the shear stress $\tau$ comes from the first derivative of potential for the whole system and the shear strain $\gamma$ is obtained lateral displacement divided by the height of the model. The critical shear stress (CSS) was $542 \mathrm{MPa}(\gamma=0.960)$ for the basal slip and $663 \mathrm{MPa}(\gamma=0.142)$ for the prism slip, respectively. The basal slip quite easily occurs due to the largest interlayer distance of $0.26 \mathrm{~nm}$. On the other hand, for the $\{10 \overline{1} 1\}\langle 11 \overline{2} 3\rangle$ first-pyramidal slip and the $\{11 \overline{2} 2\}\langle 11 \overline{2} 3\rangle$ second-pyramidal slip, the results by EAM exhibit no obvious dislocation gliding on their slip planes. For the first-pyramidal slip as shown in Fig. 2(a), many basal partial dislocation pairs were created from the center region of the model and then a fcc structure appeared at the peak in the shear stress, $704 \mathrm{MPa}$ $(\gamma=0.157)$. The atomic structure of arrayed basal partial dislocations is recognized as the deformation kink. ${ }^{14-16)}$ The deformation kink was reported in $\mathrm{Zn}^{14)}$ and $\mathrm{Cd}^{15)}$ but it has not been experimentally observed in $\mathrm{Mg}$ alloys, aside from long-period stacking ordered $\mathrm{Mg}$ alloys. ${ }^{16)}$ Although the formation of the deformation kink occurred in simulations with different model sizes of 8000 and 64000 atoms, this result would be affected by the computational conditions. Despite this discrepancy, from a qualitative point of view, it can be consider that the first-pyramidal slip is difficult to be activated. In the case of the second-pyramidal slip as shown in Figs. 2(b) and 2(c), basal and prism dislocations were initially created from the free surfaces near the corners of the model (Fig. 2(b)) and then association of these dislocations formed an inhomogeneous configuration, from which $\{10 \overline{1} 2\}$ twin arose (Fig. 2(c)) at the peak in the shear stress, $1255 \mathrm{MPa}(\gamma=0.111)$. The twin structure was confirmed by the angle between basal planes. Accordingly, the firstpyramidal slip and the second-pyramidal slip are hard to be operative, compared with the basal slip and the prism slip. However, the results by the GEAM potential differently indicate that a dislocation on the second-pyramidal slip was generated as shown in Fig. 3. In the GEAM simulation, the activation barrier of the second-pyramidal slip could be lower than the other events expected by EAM and, in result, the second-pyramidal slip was enhanced with ease. This would be related to a reduction of the second-pyramidal SFE by GEAM. As shown in Fig. 3(b), the second-pyramidal dislocation generated in the GEAM simulation was extended with the stacking fault. Sandlöbes et al. reported that pyramidal $\langle c+a\rangle$ dislocations have been observed in $\mathrm{Mg}-$ $\mathrm{Y}$ alloys and the activation of $\langle c+a\rangle$ dislocations has been attributed to Y-induced reduction of the basal SFE. ${ }^{9)} \mathrm{A}$ recent density functional theory (DFT) calculation has shown that the second-pyramidal SFE of $0.298 \mathrm{~J} / \mathrm{m}^{2}$ in pure $\mathrm{Mg}$ is reduced to $0.278 \mathrm{~J} / \mathrm{m}^{2}$ in $\mathrm{Mg}-\mathrm{Y}$ alloys. ${ }^{17)}$ Although the stacking fault of the second-pyramidal plane is complex and it is difficult to understand a detailed mechanism of the reduction of the secondary-pyramidal SFE, the present simulations by GEAM with lower SFEs of basal and secondpyramidal planes are corresponding to the $\mathrm{Mg}-\mathrm{Y}$ case.

For the shear deformation of the $\{10 \overline{1} 2\}\langle 10 \overline{1} 1\rangle$ twinning, as shown in Fig. 4, basal and first-pyramidal dislocations were initially created at $668 \mathrm{MPa}(\gamma=0.067)$ from the free surfaces near the corners of the model and then the $\{10 \overline{1} 2\}$ twins are nucleated at $724 \mathrm{MPa}(\gamma=0.109)$ from the inhomogeneous region where the dislocations were associated, which is similar to the second-pyramidal slip by EAM in Figs. 2(b) and 2(c). As increasing the shear strain, the $\{10 \overline{1} 2\}$ twins grew with a winding morphology. This corresponds to a thick lenticular $\{1012\}$ twin observed experimentally ${ }^{4}$ and is attributed to the small dislocation energy and the low Peierls barrier of the twinning dislocation of the $\{10 \overline{1} 2\}$ twin, which have been reported in our previous study. ${ }^{18)}$

Figure 5(a) shows the shear deformation of the $\{10 \overline{1} 1\}$ twinning in the direction of $\langle 10 \overline{1} \overline{2}\rangle$, which is the Burgers vector direction of the 4-layer twinning dislocation; ${ }^{19,20)}$ $b_{4}^{\{10 \overline{1} 1\}}=\frac{4 r^{2}-9}{4 r^{2}+3}\langle 10 \overline{1} \overline{2}\rangle$ where $r$ is the $c / a$ ratio. Many basal dislocations were created at the early stage of shear deformation. Further deformation provided inhomogeneous regions at the corners of the model, where a small $\{10 \overline{1} 1\}$ twin with a different orientation and a planer defect of the $\{10 \overline{1} 1\}$ plane were formed. Nucleation and growth of the appropriate $\{10 \overline{1} 1\}$ twin of which the $K_{1}$ plane is normal to the $z$-axis were not observed in our simulations. However, for the $\{10 \overline{1} 1\}$ twinning in the direction of the Burgers vector of the 2-layer twinning dislocation, $b_{2}^{\{1011\}}=\frac{1}{2} b_{4}^{\{10 \overline{1} 1\}} \pm$ $\frac{1}{6}\langle 1 \overline{2} 10\rangle,{ }^{19,20)}$ the $\{10 \overline{1} 1\}$ twin was nucleated in the center of the model at the CSS of $421 \mathrm{MPa}$, as shown in Fig. 5(b). In Fig. 5(c), the $\{10 \overline{1} 1\}$ twin immediately took a banded morphology with the coherent twin boundaries and grew by the glide of the twinning dislocations (Fig. 5(d)), consistent with the narrow banded $\{10 \overline{1} 1\}$ twin observed in Mg alloys. $\left.{ }^{4}\right)$ From the results of shear deformations on the same $\{10 \overline{1} 1\}$ plane but in different shear directions, the CSS for nucleation of the $\{10 \overline{1} 1\}$ twin has the minimum value at the direction of nearly 66 degrees with respect to $\langle 10 \overline{1} \overline{2}\rangle$, which is identified as the $b_{2}^{\{10 \overline{1} 1\}}$ direction. Therefore, for the $\{10 \overline{1} 1\}$ twinning, 


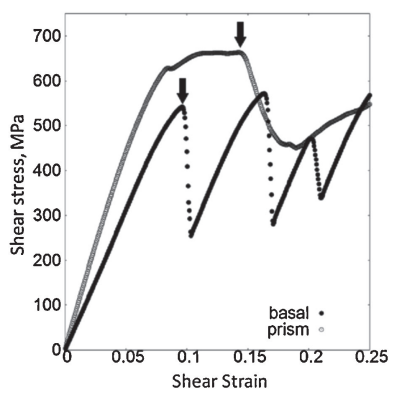

(a)

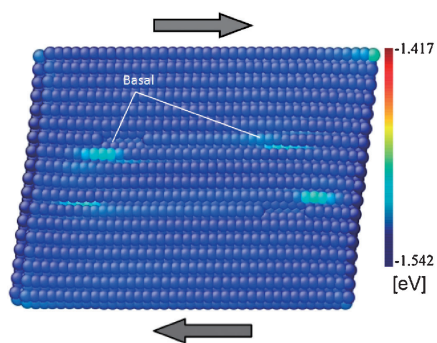

(b)

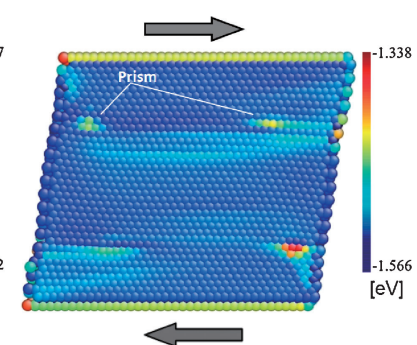

(c)
Fig. 1 (a) Shear stress as a function of shear strain for the basal slip and the prism slip. The basal and prism dislocations are activated at the CSS indicated by arrows. MD snapshots of (b) the basal dislocations at $\gamma=0.101$ and (c) the prism dislocations at $\gamma=0.154$. Atoms are colored by their excess potential energies.

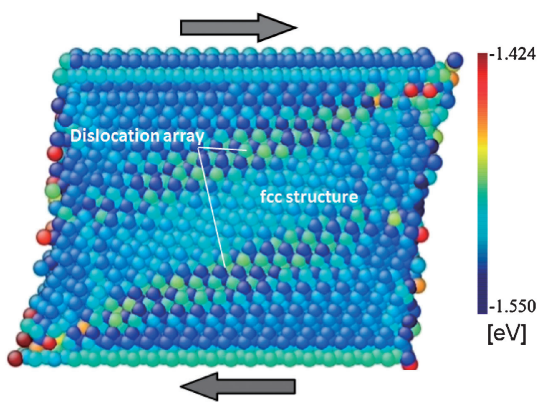

(a)

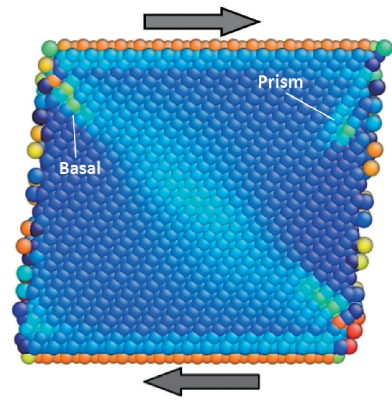

(b)

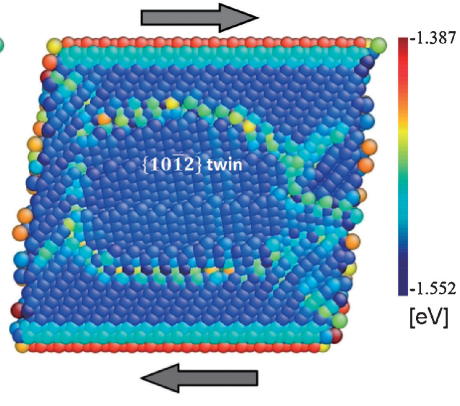

(c)
Fig. 2 Complex plastic deformations of (a) the first-pyramidal slip at $\gamma=0.160$ and the second-pyramidal slip at (b) $\gamma=0.110$ and (c) $\gamma=0.145$. Atoms are colored by their excess potential energies.

the $b_{4}^{\{10 \overline{1} 1\}}$ shear direction of $\langle 10 \overline{1} \overline{2}\rangle$ is not preferable but the $b_{2}^{\{10 \overline{1} 1\}}$ shear direction is more probable. This is because the $b_{2}^{\{1011\}}$ shear requires less atomic shuffling for the $\{10 \overline{1} 1\}$ twinning than the $b_{4}^{\{1011\}}$ one. The previous studies ${ }^{18)}$ have

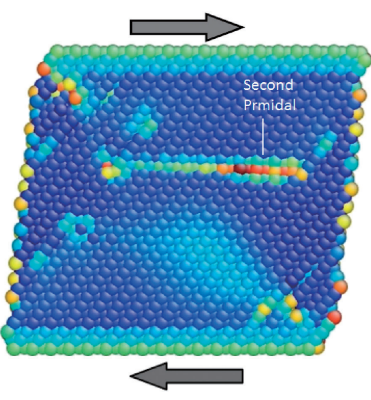

(a)

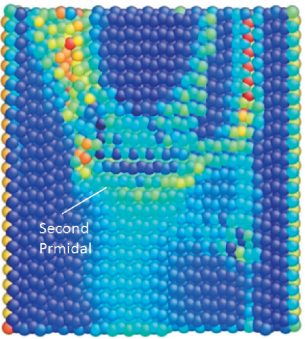

(b)
Fig. 3 Activation of the second-pyramidal slip in the shear deformation of $\{11 \overline{2} 2\}\langle 11 \overline{2} 3\rangle$ by the GEAM potential. (a) $x z$ and (b) $x y$ cross-sectional views of MD snapshot. Atoms are colored by their excess potential energies.

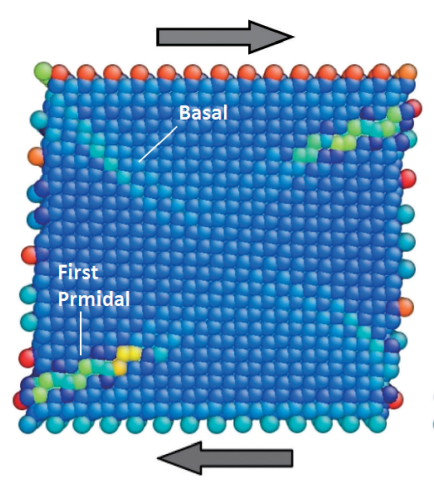

(a)

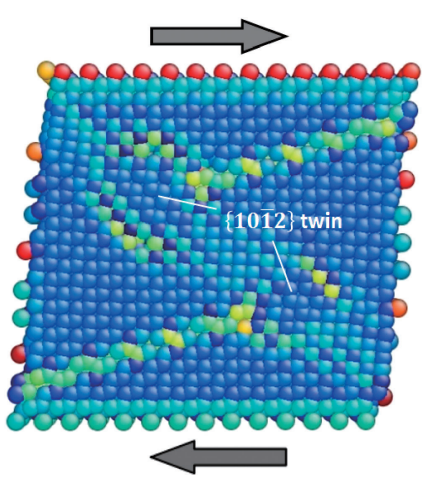

(b)

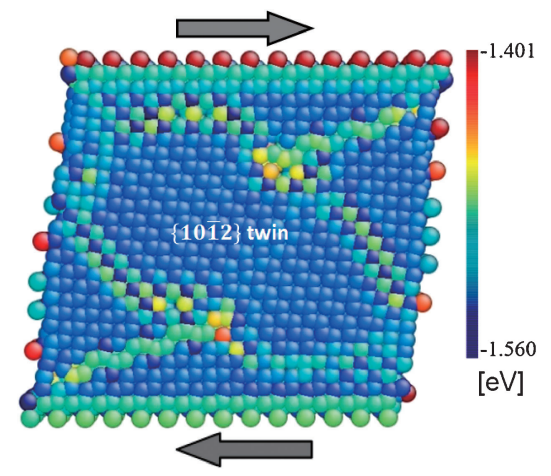

(c)

Fig. 4 Inhomogeneous nucleation and growth of the $\{10 \overline{1} 2\}$ twins on the simple shear deformation simulation for the $\{10 \overline{1} 2\}\langle 10 \overline{1} 1\rangle$ twinning. The atomic configurations at (a) $\gamma=0.075$, (b) $\gamma=0.117$ and (c) $\gamma=0.177$. Atoms are colored by their excess potential energies.

found that the Peierls barrier of the $b_{2}^{\{10 \overline{1} 1\}}$ twinning dislocation is lower than that of the $b_{4}^{\{10 \overline{1} 1\}}$ twinning dislocation, and this fact strongly supports the present results physically and energetically.

Before the $\{10 \overline{1} 1\}$ twin nucleation, a striped modulation of the excess potential energies of atoms parallel to the basal plane appeared, as shown in Fig. 5(b), which implies the basal slip has bearing on the $\{10 \overline{1} 1\}$ twinning. For the $\{10 \overline{1} 2\}\langle 10 \overline{1} 1\rangle$ twinning, uniform nucleation of the $\{10 \overline{1} 2\}$ twin is unfavorable, and association of dislocations as a precursor is necessary to generate the $\{10 \overline{1} 2\}$ twin. Uniform 


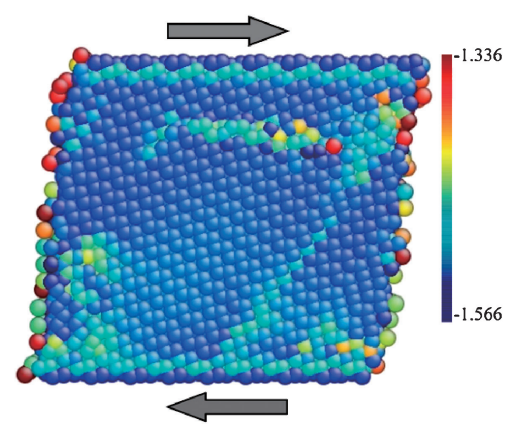

(a)

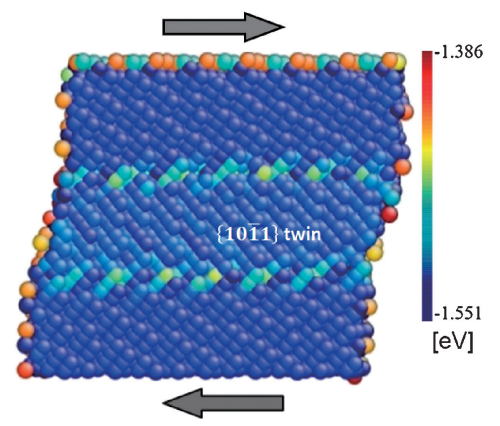

(c)

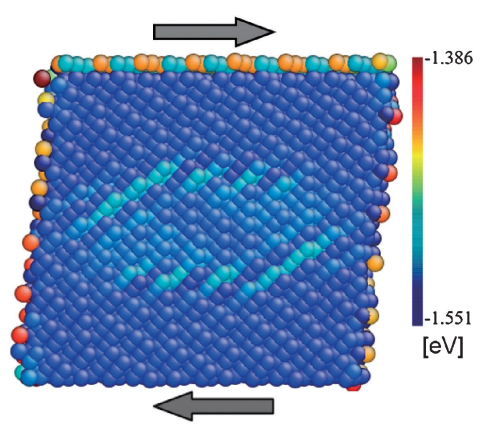

(b)

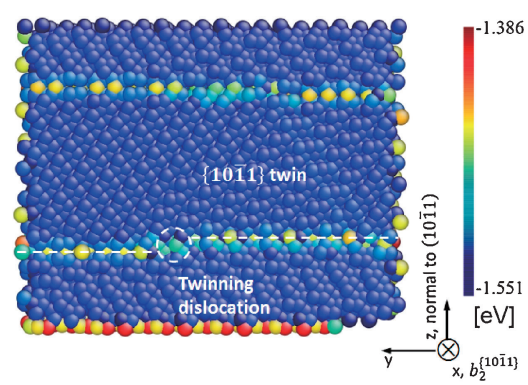

(d)

Fig. 5 Simple shear deformations with respect to the $\{10 \overline{1} 1\}$ plane, (a) in the $\langle 10 \overline{1} \overline{2}\rangle$ shear direction at $\gamma=0.160$, and in the $b_{2}^{\{10 \overline{1}\}}$ shearing direction at (b) $\gamma=0.100$, (c) $\gamma=0.130$ and (d) $\gamma=0.210$. Atoms are colored by their excess potential energies.

Table 2 The critical shear stress for fundamental deformation modes by the EAM and GEAM potentials in units of MPa. The values in parenthesis are the ratio to the basal value. For the cases that the dislocation generation or the twin nucleation is not observed, the peak shear stress is applied, which is denoted by an asterisk.

\begin{tabular}{lccccccc}
\hline & \multicolumn{3}{c}{ Slip } & \multicolumn{3}{c}{ Twinning } \\
\hline Plane & $\{0001\}$ & $\{10 \overline{1} 0\}$ & $\{10 \overline{1} 1\}$ & $\{11 \overline{2} 2\}$ & $\{10 \overline{1} 2\}$ & $\{10 \overline{1} 1\}$ & $\{10 \overline{1} 1\}$ \\
Direction & $\langle 1 \overline{2} 10\rangle$ & $\langle\overline{1} 2 \overline{1} 0\rangle$ & $\langle 11 \overline{2} 3\rangle$ & $\langle 11 \overline{2} 3\rangle$ & $\langle 10 \overline{1} 1\rangle$ & $\langle 10 \overline{1} \overline{2}\rangle$ & $b_{2}^{\{10 \overline{1} 1\}}$ \\
\hline \multirow{2}{*}{ EAM } & 542 & 663 & $703^{*}$ & $1254^{*}$ & 742 & $1398^{*}$ & 421 \\
& & $(1.22)$ & $(1.30)$ & $(2.31)$ & $(1.37)$ & $(2.58)$ & $(0.78)$ \\
\multirow{2}{*}{ GEAM } & 572 & 892 & $711^{*}$ & 1053 & 915 & $1035^{*}$ & 584 \\
& & $(1.56)$ & $(1.24)$ & $(1.84)$ & $(1.60)$ & $(1.81)$ & $(1.02)$ \\
\hline
\end{tabular}

nucleation models require substantially high stress ${ }^{3)}$ and nonuniform twin nucleation around the inhomogeneous site should be more probable. Since the basal slip quite easily occurs, the non-uniform twin nucleation by the initially created dislocations is likely, even if there is no preexisting defect ideally.

The CSS values obtained from both the potentials for the fundamental deformation modes are summarized in Table 2. Although it is difficult to directly discuss the CSS values of the fundamental deformation modes and to compare them quantitatively with the experimental CRSS values obtained so far, due to differences between the simulation conditions and actual materials, the simulation results can be discussed qualitatively. For deformation cases that generation of intentional dislocations or twins is not observed, such as for the pyramidal slips and the $\{10 \overline{1} 1\}$ twinning in the $b_{4}^{\{10 \overline{1} 1\}}$ shear direction, the peak in the shear stress is applied as the
CSS value in this study, which is denoted by an asterisk in Table 2. This means that the pyramidal slips and the $\{10 \overline{1} 1\}$ twinning in the $b_{4}^{\{1011\}}$ shear direction are hard to be activated even at the CSS, qualitatively consistent with a tendency in experiments. Based on the GEAM results, reduction of the second-pyramidal SFE activated the second-pyramidal slip as shown in Fig. 3. Additionally, the CSS of the $\{10 \overline{1} 1\}$ twinning in the $b_{2}^{\{10 \overline{1} 1\}}$ direction was increased. Although the twin boundary energies for the $\{10 \overline{1} 2\}$ and $\{10 \overline{1} 1\}$ twins by GEAM, 106 and $73 \mathrm{~mJ} / \mathrm{m}^{2}$, were lower than 113 and $106 \mathrm{~mJ} / \mathrm{m}^{2}$ by EAM respectively, the dislocation energy and the Peierls barrier of twinning dislocations are important for deformation twinning, on which effects of solute atoms will be studied in our future work. For the other fundamental deformation modes, the relative order in the CSS was qualitatively maintained despite the reduction of the basal SFE.

\section{Summary}

In the present study, we have carried out MD simulations of simple shear deformation to investigate the fundamental deformation modes of slip and deformation twinning in magnesium. By employing EAM and GEAM potentials, effects of SFEs on the fundamental deformation modes have been investigated. While the basal slip quite easily occurred and the prism dislocation was activated, the first-pyramidal slip and the second-pyramidal slip were hard to be operated. In the simulations for these pyramidal slips by EAM, pyramidal dislocations were not generated and the shear deformation gave rise to other deformation mechanisms. However, in the GEAM results with low SFEs of the basal 
and second-pyramidal planes, the second-pyramidal slip was enhanced. This is corresponding to experimental and DFT studies for $\mathrm{Mg}-\mathrm{Y}$ alloys. On the other hand, the simulations have also demonstrated that the $\{10 \overline{1} 2\}$ twin is generated from an inhomogeneous structure due to association of dislocations. For the $\{10 \overline{1} 1\}$ twinning, the $\langle 10 \overline{1} \overline{2}\rangle$ shear direction was not preferable and the $\{10 \overline{1} 1\}$ twin was nucleated uniformly in the $b_{2}^{\{1011\}}$ shearing direction. In addition to the enhancement of the second-pyramidal slip, the uniform nucleation of the $\{10 \overline{1} 1\}$ twin in the $b_{2}^{\{10 \overline{1} 1\}}$ shearing direction was suppressed in the GEAM simulation. Meanwhile, the relative order of the other fundamental deformation modes in the CSS is qualitatively maintained despite the reduction of the SFEs.

\section{Acknowledgments}

This work has been supported by the Toyota Initiative on Magnesium alloys.

\section{REFERENCES}

1) S. R. Agnew and J. F. Nie: Scr. Mater. 63 (2010) 671.

2) M. H. Yoo: Metall. Trans. A 12 (1981) 409.

3) J. W. Christian and S. Mahajant: Prog. Mater. Sci. 39 (1995) 1.
4) J. Koike: Metall. Mater. Trans. A 36 (2005) 1689.

5) R. E. Reed-Hill and H. D. Robertson: Acta Metall. 5 (1957) 717.

6) N. Stanford, D. Atwell and M. R. Barnett: Acta Mater. 58 (2010) 6773.

7) S. R. Agnew, M. H. Yoo and C. N. Tomé: Acta Mater. 49 (2001) 4277.

8) Y. Chino, M. Kado and M. Mabuchi: Acta Mater. 56 (2008) 387.

9) S. Sandlöbes, M. Friák, S. Zaefferer, A. Dick, S. Yi, D. Letzig, Z. Pei, L.-F. Zhu, J. Neugebauer and D. Raabe: Acta Mater. 60 (2012) 3011.

10) D. Y. Sun, M. I. Mendelev, C. A. Becker, K. Kudin, T. Haxhimali, M. Asta, J. J. Hoyt, A. Karma and D. J. Srolovitz: Phys. Rev. B 73 (2006) 024116.

11) X. W. Zhou, R. A. Johnson and H. N. G. Wadley: Phys. Rev. B 69 (2004) 144113.

12) W. F. Gale and T. C. Totemeier: Smithells Metals Reference Book, Eighth Edition, (Elsevier Inc., 2004).

13) A. Couret and D. Caillard: Acta Metall. 33 (1985) 1455.

14) J. B. Hess and C. S. Barrett: Trans. Am. Inst. Min. Met. Eng. 185 (1949) 599.

15) E. Orowan: Nature 149 (1942) 643.

16) K. Hagihara, N. Yokotani and Y. Umakoshi: Intermetallics 18 (2010) 267.

17) Z. Pei, L.-F. Zhu, M. Friák, S. Sandlöbes, J. von Pezold, H. W. Sheng, C. P. Zaefferer, B. Svendsen, D. Raabe and J. Neugebauer: New J. Phys. 15 (2013) 043020.

18) D. Matsunaka, A. Kanoh and Y. Shibutani: Mater. Trans. 54 (2013) 1524-1527.

19) A. Serra, R. C. Pond and D. J. Bacon: Acta Metall. Mater. 39 (1991) 1469.

20) J. Wang, I. J. Beyerlein, J. P. Hirth and C. N. Tomé: Acta Mater. 59 (2011) 3990 . 